\title{
Steady two-dimensional free-surface flow over semi- infinite and finite-length corrugations in an open channel
}

DOI:

10.1103/PhysRevFluids.3.114804

\section{Document Version}

Final published version

Link to publication record in Manchester Research Explorer

\section{Citation for published version (APA):}

Keeler, J., Binder, B., \& Blyth, M. (2018). Steady two-dimensional free-surface flow over semi-infinite and finitelength corrugations in an open channel. Physical Review Fluids, 1-11. [114804].

https://doi.org/10.1103/PhysRevFluids.3.114804

\section{Published in:}

Physical Review Fluids

\section{Citing this paper}

Please note that where the full-text provided on Manchester Research Explorer is the Author Accepted Manuscript or Proof version this may differ from the final Published version. If citing, it is advised that you check and use the publisher's definitive version.

\section{General rights}

Copyright and moral rights for the publications made accessible in the Research Explorer are retained by the authors and/or other copyright owners and it is a condition of accessing publications that users recognise and abide by the legal requirements associated with these rights.

\section{Takedown policy}

If you believe that this document breaches copyright please refer to the University of Manchester's Takedown Procedures [http://man.ac.uk/04Y6Bo] or contact uml.scholarlycommunications@manchester.ac.uk providing relevant details, so we can investigate your claim.

\section{OPEN ACCESS}




\title{
Steady two-dimensional free-surface flow over semi-infinite and finite-length corrugations in an open channel
}

\author{
Jack S. Keeler \\ School of Mathematics, University of Manchester, United Kingdom \\ Benjamin J. Binder ${ }^{\dagger}$ \\ School of Mathematical Sciences, University of Adelaide, South Australia, Australia \\ Mark G. Blyth ${ }^{\ddagger}$ \\ School of Mathematics, University of East Anglia, United Kingdom \\ (Received 13 June 2018; published 26 November 2018)

\begin{abstract}
Free-surface flow past a semi-infinite or a finite-length corrugation in an otherwise flat and horizontal open channel is considered. Numerical solutions for the steady flow problem are computed using both a weakly nonlinear and fully nonlinear model. The new solutions are classified in terms of a depth-based Froude number and the four classical flow types (supercritical, subcritical, generalized hydraulic rise, and hydraulic rise) for flow over a small bump. While there is no hydraulic fall solution for semi-infinite topography, we provide strong numerical evidence that such a solution does exist in the case of a finite-length corrugation. Numerical solutions are also found for the other flow types for either semi-infinite or finite-length corrugation. For subcritical flow over a semi-infinite corrugation, the free-surface profile is found to be quasiperiodic downstream.
\end{abstract}

DOI: 10.1103/PhysRevFluids.3.114804

\section{INTRODUCTION}

Corrugated topography naturally arises in the flow of water over erodible beds in tidal estuaries, beaches, streams, and rivers [1-6]. Such flows have been studied experimentally using engineered corrugated topographies in rectangular open channels [7-11]. An important finding was that including a corrugation over part of the channel can provoke a hydraulic rise, which assists in slowing down the stream velocity and also enhances energy dissipation. Both of these outcomes may be advantageous in applications where it is desired to protect hydraulic infrastructures such as weirs, spillways, and gates [7-11]. Flow past corrugated topography is therefore an important problem for study.

In this work we investigate steady potential flow past semi-infinite and finite-length corrugations with a particular focus on computing the shape of the free surface. This extends the work of Binder et al. [12], who determined the free-surface response in the case of an infinite corrugation. We assume that there is uniform flow far upstream where the topography becomes flat (see Fig. 1). Under these conditions the radiation condition is satisfied, and the flow can be characterized by the

\footnotetext{
*jack.keeler@manchester.ac.uk

†benjamin.binder@adelaide.edu.au

${ }^{\ddagger}$ mark.blyth@uea.ac.uk
} 


\section{Sketch of semi-infinite corrugation flow problem}

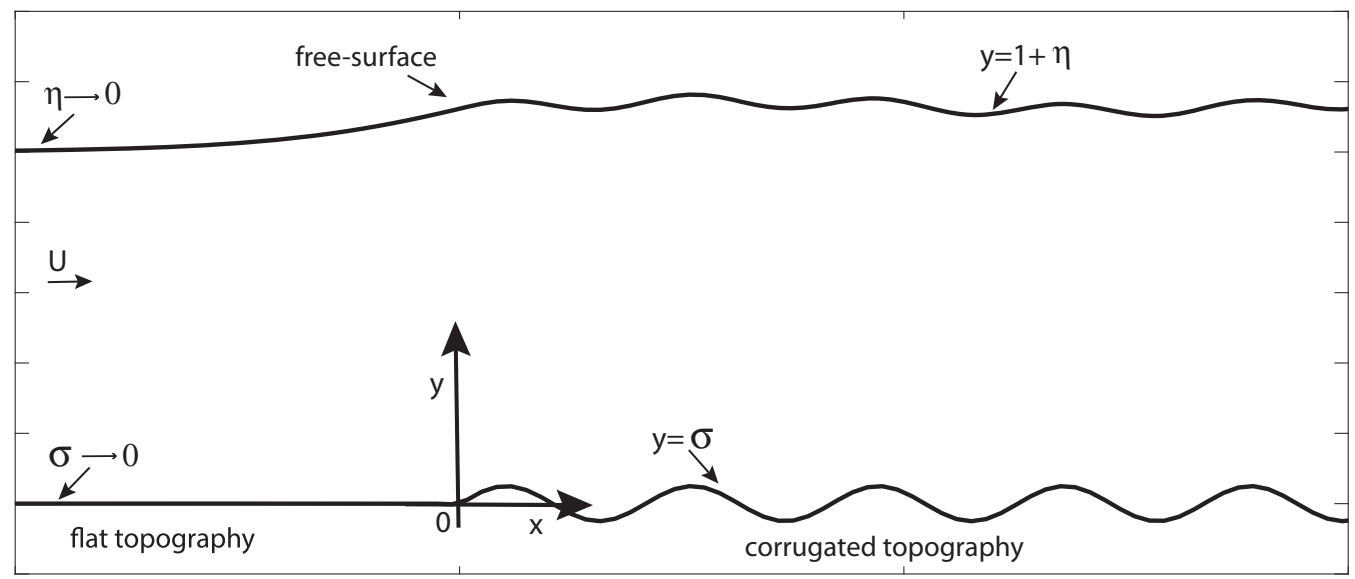

FIG. 1. Sketch of semi-infinite corrugation flow problem. The flow is from left to right with uniform flow as $x \rightarrow-\infty$.

upstream depth-based Froude number

$$
F=\frac{U}{\sqrt{g H}}
$$

where $U$ and $H$ are the constant velocity and depth far upstream, respectively, and $g$ is the acceleration due to gravity. We work assuming that quantities have been made dimensionless using $H$ as the length scale and $U$ as the velocity scale. The location of the free surface is given by $y=1+\eta(x)$, where $\eta$ is the elevation of the free surface above the constant unit depth far upstream, and the topography is denoted by $y=\sigma(x)$.

Both weakly nonlinear and fully nonlinear solutions to the problem are classified using the Froude number, $F$, and the qualitative flow features far downstream as $x \rightarrow \infty$. Specifically, to classify our results we generalize the descriptions for the classical problem of flow over a small bump for which there are four basic flow types for $F \neq 1$ (see Fig. 2). For the semi-infinite topography depicted in Fig. 1 the free surface is not expected to be flat downstream even in the
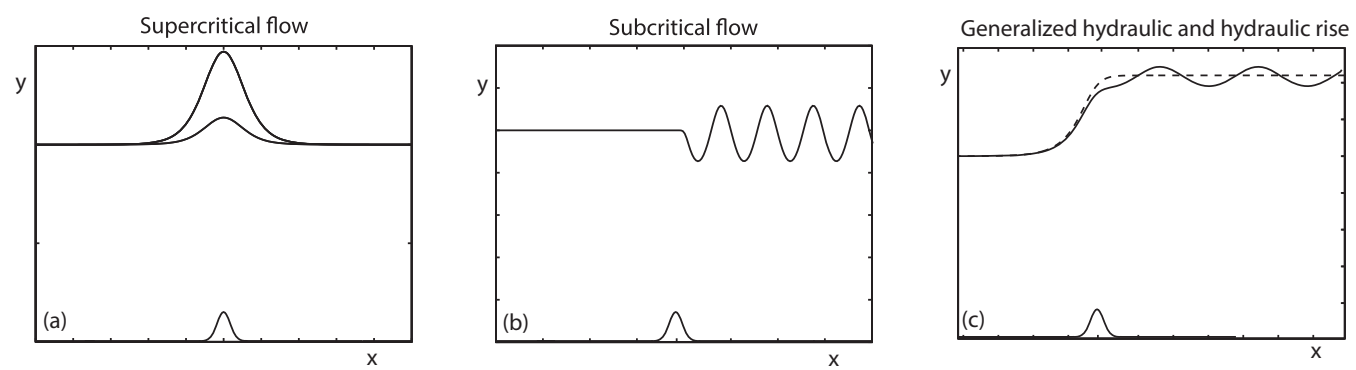

FIG. 2. Sketch of the four basic flow types for flow over a bump. (a) Supercritical flow. The solutions are nonunique with two types of solution for a given value of $F>1$ and a given bump. The solution with the largest value of $\eta$ is called a perturbation of a solitary wave. The other solution is referred to as a perturbation of a uniform stream. (b) Subcritical flow. The solution is unique for a given value of $F<1$ and a given bump. (c) Generalized hydraulic rise (solid curve). There is an infinite number of solutions for a given value of $F>1$ and a given bump. Hydraulic rise (broken curve). There is a unique solution for a given bump; the Froude number, $F>1$, cannot be chosen independently and comes as part of the solution. 
case of supercritical flow. Nevertheless it will be helpful to attribute to it a characteristic elevation; for example, if the solution approaches a periodic state far downstream, this might be taken to be the average value of $\eta$ over one wavelength. The four flow types used to categorize our results are described as follows.

(1) Supercritical flow with $F>1$ and a characteristic free-surface elevation of $\eta=0$ as $x \rightarrow \infty$ $[13,14]$.

(2) Subcritical flow with $F<1$ and a characteristic free-surface elevation of $\eta=0$ as $x \rightarrow \infty$ $[15,16]$.

(3) Generalized hydraulic rise with $F>1$ and a characteristic free-surface elevation of $\eta>0$ as $x \rightarrow \infty$ [17].

(4) Hydraulic rise with $F>1$, and uniform flow with a characteristic free-surface elevation of $\eta>0$ as $x \rightarrow \infty[14,18]$.

Note that because of the reversibility of the flow types 3 and 4 may also be interpreted as hydraulic falls $[19,20]$. We calculate steady solutions corresponding to flow types $1-3$ for both semiinfinite and finite-length corrugation, but are able to find solutions only of type 4 for finite-length corrugation.

\section{FORMULATION}

We consider the steady, two-dimensional, irrotational, inviscid, and incompressible fluid flow over both semi-infinite and finite-length corrugated topography. The semi-infinite corrugated topography is given by

$$
\sigma(x)=\frac{\epsilon}{2} \cos (k x)[1+\tanh (\lambda x)]
$$

where $\epsilon$ and $k$ correspond to the amplitude and wave number of the corrugation, and $\lambda$ is a constant. The finite-length corrugation is taken to be of the form

$$
\sigma(x)=\frac{\epsilon}{2} \cos (k x)\{\tanh [\lambda(x+\gamma)]-\tanh [\lambda(x-\gamma)]\},
$$

where $\gamma$ is a measure of the length of the corrugation. The boundary conditions far upstream for the semi-infinite and finite-length corrugation are

$$
\eta \rightarrow 0 \text { and } \eta_{x} \rightarrow 0 \quad \text { as } \quad x \rightarrow-\infty
$$

Further details on the boundary conditions will be given in Sec. IV.

Written in terms of the velocity potential $\phi$, the fully nonlinear problem is to solve Laplace's equation

$$
\nabla^{2} \phi=0 \quad \text { in } \quad-\infty<x<\infty, \quad \sigma(x)<y<1+\eta(x),
$$

subject to the dynamic and kinematic boundary conditions on the free surface,

$$
\left.\begin{array}{l}
\frac{1}{2}|\nabla \phi|^{2}+\frac{1}{F^{2}} y=\frac{1}{2}+\frac{1}{F^{2}} \\
\eta_{x} \phi_{x}=\phi_{y}
\end{array}\right\} \quad \text { on } \quad y=1+\eta(x)
$$

along with the no-penetration condition on the bottom,

$$
\sigma_{x} \phi_{x}=\phi_{y} \quad \text { on } \quad y=\sigma(x),
$$

and the far-field conditions (4) together with $\phi \rightarrow x$ as $x \rightarrow-\infty$.

In the limit of small-amplitude waves Refs. [21-25] showed that the fully nonlinear problem (5)-(7) reduces to the forced Korteweg-de Vries equation (fKdV),

$$
\eta_{x x}+\frac{9}{2} \eta^{2}-6(F-1) \eta=-3 \sigma(x)
$$


The advantage of using this model equation over the full system is that a much more expansive exploration of the possible solution space is practical within a reasonable computational time frame. For the most part we present solutions obtained by solving the fKdV equation numerically using finite differences and Newton iterations. These weakly nonlinear results are complemented by a few fully nonlinear calculations for comparison. The fully nonlinear computations are done numerically using the boundary integral method of Refs. [26,27] and others. With this method the dimensionless quantities are given parametrically in terms of $\phi$. As long as the amplitude of the corrugation is not too large, as will be assumed here, the flow is close to a uniform stream and so $\phi \approx x$. In this case it is more convenient to prescribe $\sigma$ as a function of $\phi$ instead of $x$. It has been found that doing this makes a minimal difference to the results [28].

\section{LINEARIZED ANALYSIS}

It is illuminating to consider the solutions of the $\mathrm{fKdV}$ equation when the amplitude of the topography is small. Working on this basis we assume that $\epsilon \ll 1$ and seek a solution to (8) in the form $\eta(x)=\epsilon N(x)$, where $N=O(1)$. Substituting into (8) and taking the limit as $\epsilon$ tends to zero, we obtain the linearized form

$$
\eta_{x x} \pm \mu^{2} \eta=-3 \sigma(x)
$$

where we have written $F=1 \pm \mu^{2} / 6$ for constant $\mu$, and where the \pm sign refers to sub- or supercritical flow, respectively. We focus on the case of a semi-infinite corrugation, and for the sake of the present analysis we take in place of (2)

$$
\sigma(x)=\epsilon H(x) \cos (k x) .
$$

For subcritical flow the solution to (9) which satisfies condition (4) is

$$
\eta=-\frac{3 \epsilon}{\left(\mu^{2}-k^{2}\right)}[\cos (k x)-\cos (\mu x)] \quad \text { if } \quad x>0 ; \quad \eta=0 \text { if } x \leqslant 0 .
$$

Evidently for a bounded solution we must insist that $\mu \neq k$ so that there is no resonance between the wall forcing frequency, $k$, and the natural frequency of the linearized system, $\mu$. For supercritical flow the solution to (9) which satisfies $\eta \rightarrow 0$ as $x \rightarrow-\infty$, and which is bounded as $x \rightarrow \infty$, is

$$
\eta=\frac{3 \epsilon}{k^{2}+\mu^{2}}\left[\cos (k x)-\frac{1}{2} \mathrm{e}^{-\mu x}\right] \quad \text { if } \quad x>0 ; \quad \eta=\frac{3 \epsilon}{2\left(k^{2}+\mu^{2}\right)} \mathrm{e}^{\mu x} \quad \text { if } \quad x \leqslant 0 .
$$

As $x \rightarrow \infty$ this matches to the small amplitude periodic solution obtained for an infinite sinusoidal corrugation by Ref. [12]. The comparison between the linearized and weakly nonlinear results will be examined in the next section.

\section{NUMERICAL RESULTS}

The manner in which the boundary conditions are imposed in our numerical schemes for both the fKdV equation (8) and the fully nonlinear system (5)-(7) differs between the subcritical flow case and the supercritical flow case. In either case we must truncate the domain. For subcritical flow we set $\eta=\eta_{x}=0$ at the upstream truncation point. This ensures that no small amplitude cnoidal waves are picked up upstream and the surface profile tends to be flat. We then solve as an initial value problem, integrating (8) forwards in $x$ from the upstream truncation point. For supercritical flow, we set $\eta=0$ at the upstream truncation point. As we require that $\eta$ remains bounded as $x \rightarrow \infty$ we truncate the problem on a domain sufficiently wide and demand that the free surface reaches a local maximum at the downstream truncation point, which itself is found as part of the solution. In practice by taking the downstream truncation point at a sufficiently large value of $x$ we find that the local maximum on the free surface almost coincides with a local maximum on the topography in line with the linearized solution (12). 

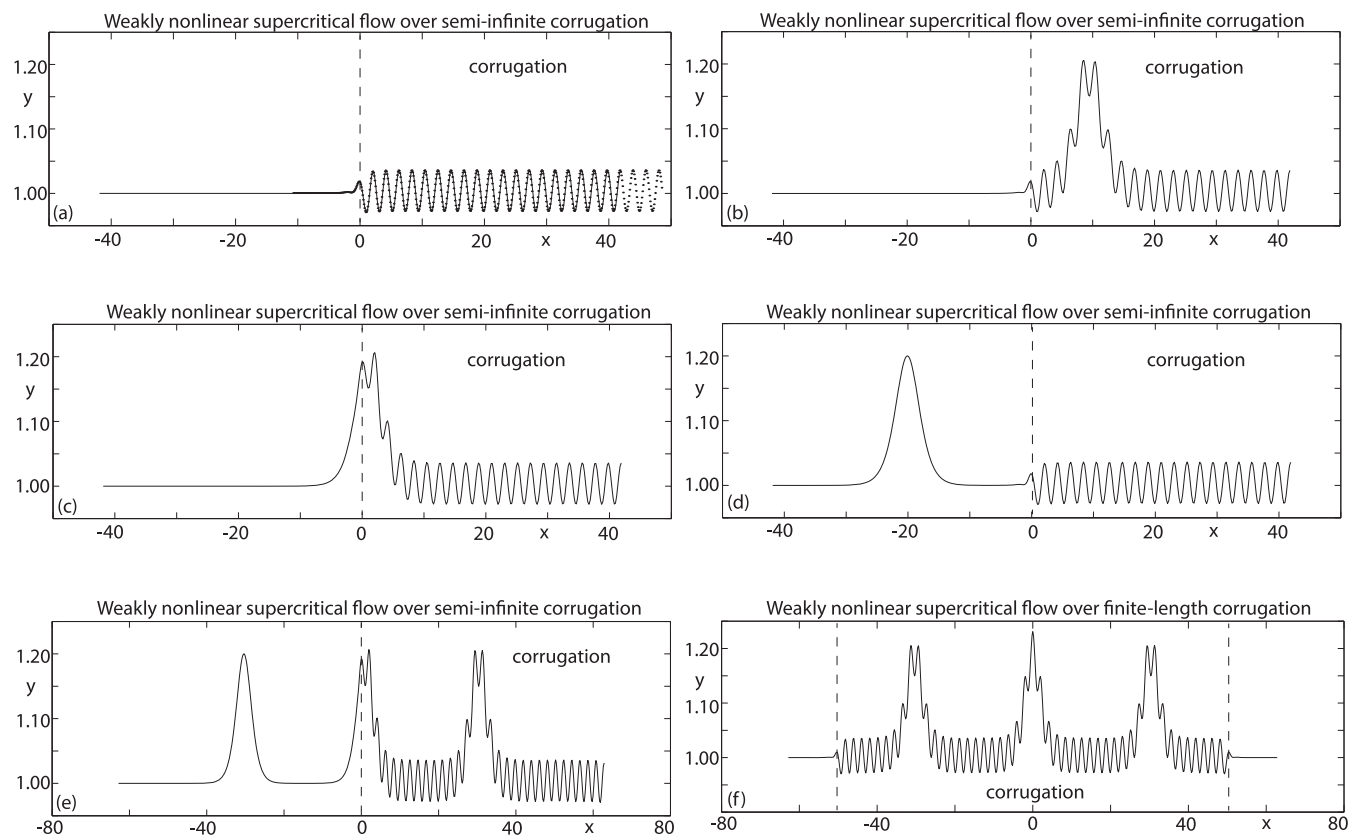

FIG. 3. The solid curves are weakly nonlinear solutions for supercritical flow with $F=1.10, \epsilon=0.1$, $k=3$, and $\lambda=1$. (a)-(e) Semi-infinite corrugation. (f) Finite-length corrugation, $\gamma=50$. The dotted curve in panel (a) is the linearized solution (12).

We compute both weakly nonlinear and fully nonlinear solutions for flow past semi-infinite and finite-length corrugations. Only the response of the free surface is shown in the following profiles, with a point of transition from a corrugated section to a flat section of the topography indicated by a vertical broken line. By necessity the surface profiles are shown on a scale where the oscillations appear to be high frequency but in fact are slowly varying, and this should be borne in mind when interpreting the figures. The solutions are classified using the flow types $1-4$. We begin our discussion with flow type 1 , or supercritical flow.

\section{A. Supercritical flow}

Figure 3(a) shows a weakly nonlinear solution for the semi-infinite corrugation (solid curve) with the linearized approximation (12) superimposed (dotted curve). As $\epsilon$ decreases the agreement between the two solutions improves. This solution can be viewed as a perturbation of a uniform stream since, if we were to incrementally reduce $\epsilon$, the free surface profile would continuously approach that for a uniform flow, i.e., it would become flat. In contrast, the solution shown in Fig. 3(b) we classify as a perturbation of a solitary wave because in this case as $\epsilon$ is incrementally reduced to zero we recover an unforced solitary wave. The perturbation of a solitary wave solution is not unique; indeed, in Figs. 3(b)-3(e) the solitary wave part of the profile is located in different places, and, moreover, in Fig. 3(e) there is more than one solitary wave part. These results are broadly similar to those found by Binder et al. [12] for an infinite corrugation, and the nonuniqueness can be explained as follows. For flat topography there are an uncountable number of solitary waves which can be obtained by taking arbitrary shifts, $x_{s}$, of the well-known free solitary wave solution

$$
\eta(x)=2(F-1) \operatorname{sech}^{2}\left[\sqrt{\frac{3(F-1)}{2}}\left(x-x_{s}\right)\right] .
$$



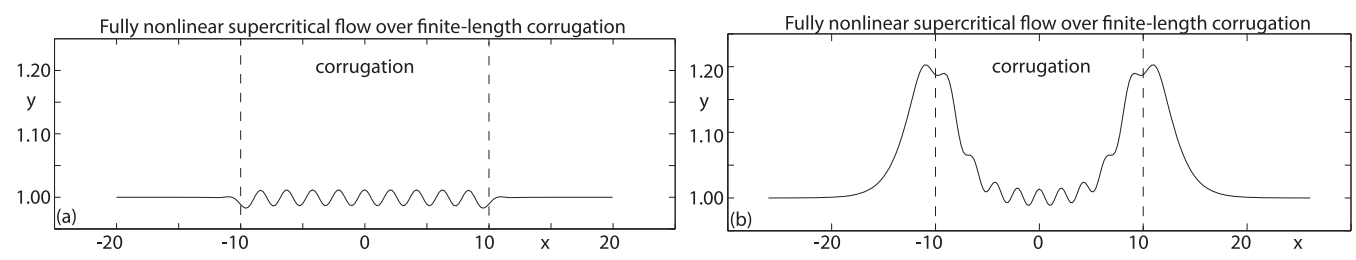

FIG. 4. Fully nonlinear solutions for supercritical flow over finite-length corrugation with $F=1.10, \epsilon=$ $0.1 k=3, \lambda=1$, and $\gamma=10$.

For corrugated topography the nonautonomous theory of Balasuriya et al. [29] can be adapted to characterise the solitary waves. As shown in Binder et al. [12], there are exactly two possible types of solitary waves on the corrugated portion of the topography: (1) those centered around crests and (2) those centered around troughs of the topography. Solitary waves within each of these two families are simple shifts of each other by multiples of $k \pi$, and therefore there are only a countable number of such solitary wave solutions.

In practice we use the form given in (13) as an initial guess in the computations. Figures 3(b)-3(d) show typical one-humped solitary wave solutions for increasing values of $x_{s}$. Furthermore, multiplehumped solitary wave solutions can be obtained in both the case of semi-infinite and finite-length topography by taking a linear combination of the form given in (13) with different values of $x_{s}$ as the initial guess in the numerical computations [e.g., see Figs. 3(e) and 3(f)]. Similar results are found in the fully nonlinear problem (e.g., see Keeler [30]). A sample case is shown in Fig. 4.

It is worth contrasting these results with those found for a bump sketched in Fig. 2(a), where the solitary wave part of the solution is located immediately above the bump.

\section{B. Subcritical flow}

Our findings indicate that for a fixed set of parameter values the solutions for subcritical flow are unique. This is in line with what is found for a bump in Fig. 2(b).

In the case of a semi-infinite corrugation, for fixed Froude number and corrugation amplitude, the effect of decreasing the wavelength of the corrugation is examined for the weakly nonlinear model in Figs. 5(a)-5(d). The dotted curve in Fig. 5(a) is the linearized approximation (11). In general there are irregular waves on the downstream free surface which become more regular as the value of $k$ is increased (in fact, all of the solutions become quasiperiodic downstream as will be discussed
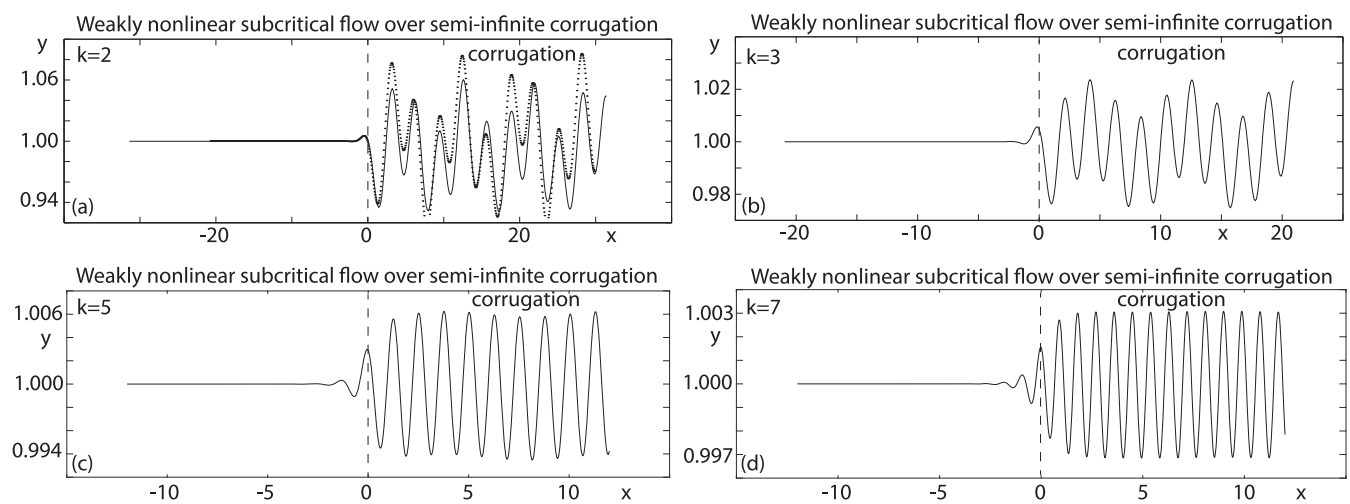

FIG. 5. The solid curves are weakly nonlinear solutions for subcritical flow with $F=0.90, \epsilon=0.05$, and $\lambda=1$. (a)-(d) Semi-infinite corrugation with (a) $k=2$, (b) $k=3$, (c) $k=5$, (d) $k=7$. The dotted curve in panel (a) is the linearized solution (11). 

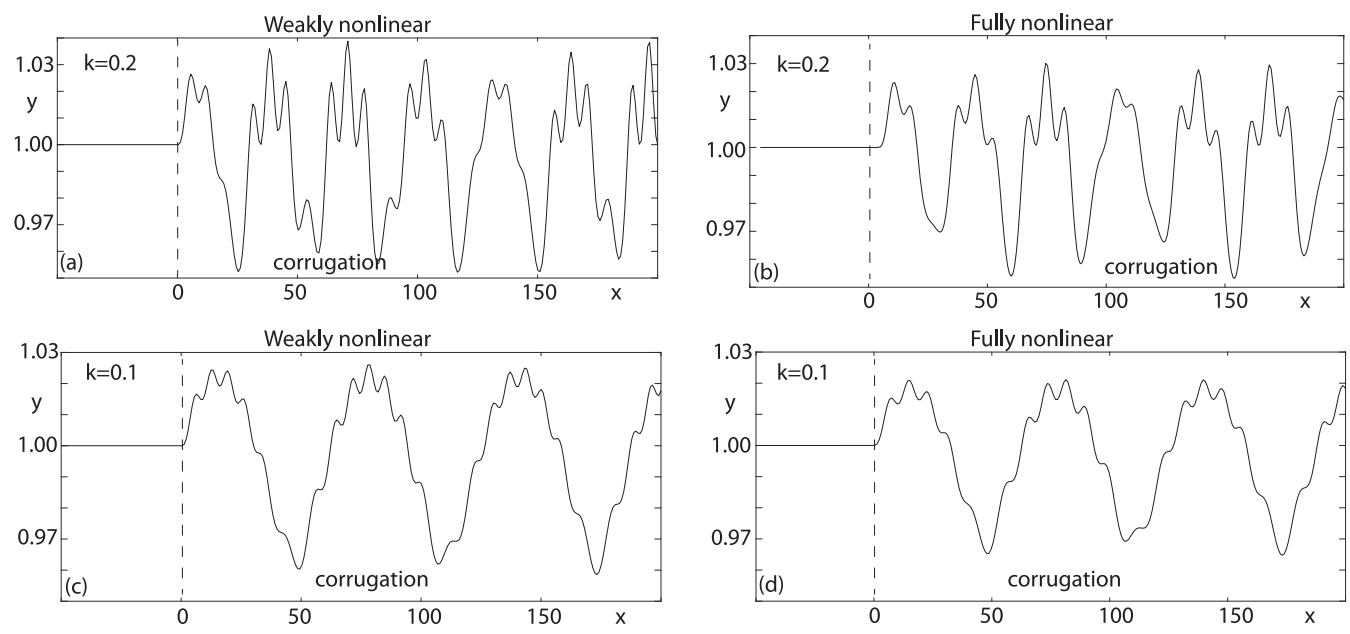

FIG. 6. Subcritical flow solutions for a semi-infinite corrugation. Comparison between weakly (a), (c) and fully nonlinear (b), (d) results for $F=0.90, \epsilon=0.005, \lambda=10$. (a, b) $k=0.2$; (c, d) $k=0.1$.

below). The same qualitative behavior is found for the fully nonlinear problem, as is illustrated in Fig. 6. This is different from what is found for a bump where only regular, periodic waves are observed downstream on the free surface.

To further examine these features in the weakly nonlinear problem for a semi-infinite corrugation, we reformulate Eq. (8) as the first-order system

$$
\left(\eta, \eta_{x}\right)_{x}=\left(\eta_{x},-3 \sigma-(9 / 2) \eta^{2}+6(F-1) \eta\right),
$$

and integrate numerically backwards in $x$ starting from the condition $\left(\eta, \eta_{x}\right)=(0,0)$. The numerical integration is done using the fourth order Runge-Kutta method with a fixed step length. As diagnostic tools, in addition to the wave profile we also plotted Poincaré sections in the $\left(\eta, \eta_{x}\right)$ plane by strobing every $2 \pi / k$ units in $x$ up to a large and negative value (typically $-10^{4}$ ), as well as constructing power spectra for the wave profiles.

The power spectra exhibit peaks at the forcing frequency $k$ and at the natural frequency of the unforced system $\sqrt{6(1-F)}$. In general these two frequencies are incommensurate, and, consequently, we expect the solutions to be quasiperiodic. This is supported by the computed Poincaré sections. For example, the Poincaré sections associated with the wave profiles in Figs. 6(a) and 6(c) are found to resemble closed loops, a classic hallmark of a quasiperiodic state (see, for example, Strogatz [31]). The strong resemblance between the weakly nonlinear results and the fully nonlinear results in Fig. 6 suggests that the fully nonlinear solutions will also be quasiperiodic downstream.

Figure 7 shows exemplar weakly nonlinear results for a finite-length corrugation. An irregular packet of waves is trapped over the topography. For a general value of the corrugation length $\gamma$ the
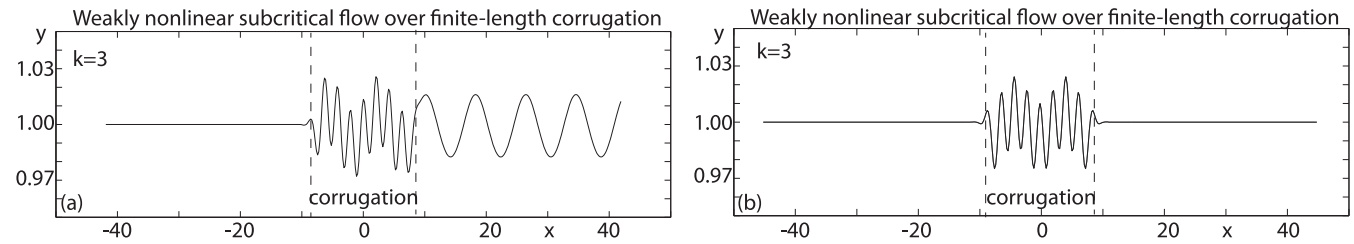

FIG. 7. Weakly nonlinear solutions for subcritical flow over a finite-length corrugation with $k=3$ for $F=0.90, \epsilon=0.05$, and $\lambda=1$. (a) $\gamma=8$, (b) $\gamma=8.42$. 

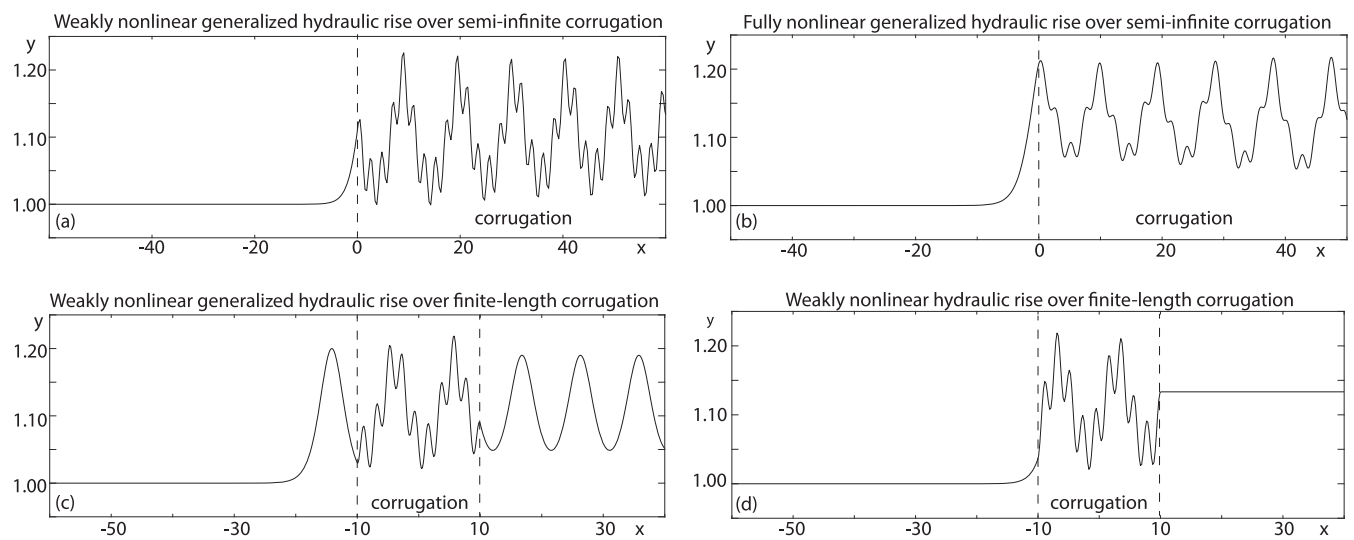

FIG. 8. Generalized hydraulic and hydraulic rise with $F=1.10, \epsilon=0.1$, and $k=3$. (a) Weakly nonlinear generalized hydraulic rise over a semi-infinite corrugation with $\lambda=10$. (b) Fully nonlinear generalized hydraulic rise over a semi-infinite corrugation with $\lambda=1$. (c) Weakly nonlinear generalized hydraulic rise over a finite-length corrugation with $\gamma=10$ and $\lambda=10$. (d) Weakly nonlinear hydraulic rise over a finite-length corrugation, $\gamma=10$ and $\lambda=10$.

downstream profile (over the flat part of the topography) is regular and periodic, as can be seen, for example, in Fig. 7(a). However, for a discrete set of $\gamma$ values (which can be found by trial and error) the downstream surface is flat. One example is shown in Fig. 7(b). Similar results, but with regular trapped waves, are seen for flows over multiple bumps [20,32] and in electrified flows [33].

\section{Hydraulic rise and generalized hydraulic rise}

For a semi-infinite topography the free surface cannot be flat downstream, and so flow type 4, namely, a hydraulic rise, is not possible. Indeed, it seems reasonable to suppose that there exist minimum and maximum downstream free surface wave amplitudes which can be attained on a given corrugation. In Figs. 8(a) and 8(b) we present generalized hydraulic rises (flow type 3) for the weakly nonlinear and fully nonlinear models. It is important to emphasize that for a given corrugation (fixed $k, \epsilon$ ) and for a fixed Froude number, $F$, there is an infinite family of generalized hydraulic rise solutions, each with a different wave profile. This is similar to the case of flow over a bump [17] apart from the important distinction that it is possible to have a hydraulic rise for a bump.

For a finite-length corrugation both generalized hydraulic and hydraulic rises are possible. A generalized hydraulic rise is shown in Fig. 8(c). In contrast to the case of a semi-infinite corrugation, the downstream wave train is regular and periodic. By demanding that the free surface is flat downstream, it is possible to obtain a hydraulic rise. An example is shown in Fig. 8(d).

\section{Hybrid solutions}

Solutions of the different flow types can be combined to construct new solutions, which are referred to as hybrid solutions [32,34]. These match two or more different flow types 1-4 in the same flow. In Fig. 9(a) we show a matching between flow types 1 and 3 (viewed as a whole, the flow is overall of type 3). Figure 9(b) matches a flow of type 1 and a flow of type 4 (globally the flow is of type 4). In both Figs. 9(c) and 9(d) there is a matching between flow type 3, a generalized hydraulic fall, and a generalized hydraulic rise, which can be viewed as a flow type 3 with a reversal of the flow direction (viewed globally, this flow is of type 1). The profiles in Figs. 9(c) and 9(d) are also referred to as table-top solitons (for this terminology see, for example, Ref. [35], which considered flow over two bumps). 

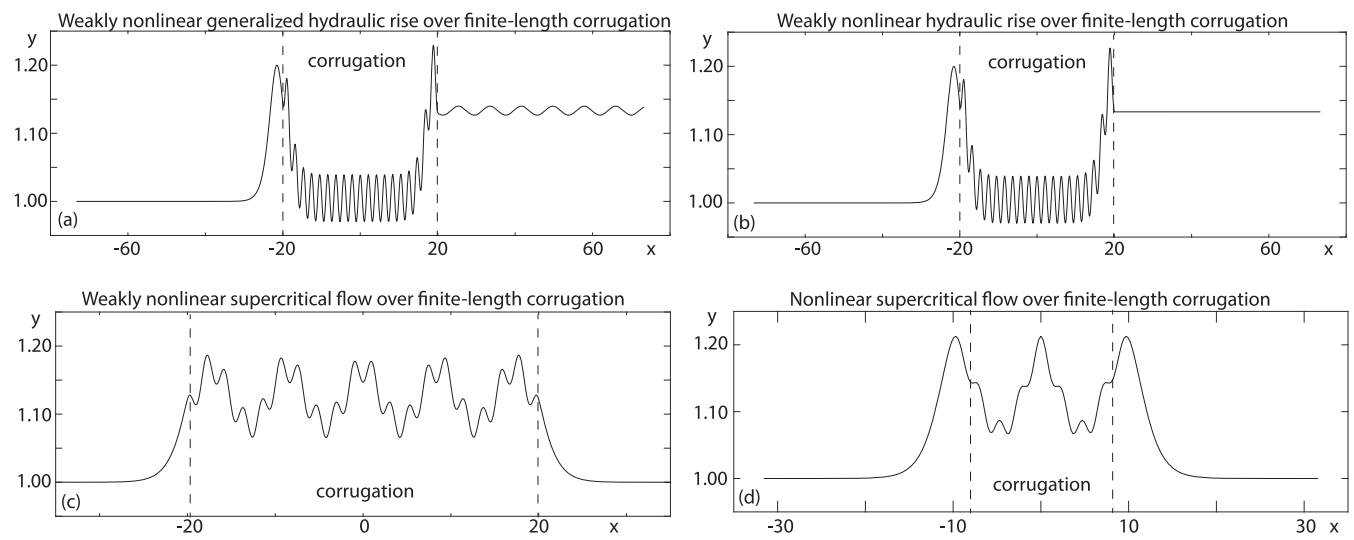

FIG. 9. Hybrid solutions on a finite-length corrugation with $F=1.10, \epsilon=0.1, k=3$, and $\lambda=10$. (a)-(c) Weakly nonlinear solutions with (a) a match between flow types 1 and 3 for $\gamma=20$ (globally the flow is type 3), (b) a match types 1 and 4 for $\gamma=20$ (globally the flow is type 4), and (c) a match between a type 3 generalized hydraulic rise and a type 3 generalized hydraulic fall for $\gamma=20$ (globally the flow is type 1). (d) Fully nonlinear solution for $\gamma=8, \lambda=1$ showing a type 3 -type 3 match as in panel (c).

\section{DISCUSSION}

We have examined steady free-surface flow over corrugated topography with a particular emphasis on semi-infinite and finite-length corrugations. We have categorized our solutions into four basic flow types 1-4 and have compared the results with the case of flow over a bump. We have also found hybrid solutions which are combinations of these flow types. Our main results are, first, that it is possible to compute type 1-3 solutions for both semi-infinite and finite-length corrugations, but type 4 solutions are possible only for corrugations of finite length, and, second, that in general subcritical flow over semi-infinite topography will pick up a quasiperiodic solution downstream and not one of the periodic solutions found in Ref. [12]. Last, for supercritical flow over a corrugated topography there is one solution which can be viewed as a perturbation of a uniform stream but an infinite number of solutions which can be categorized as perturbations of a solitary wave. In contrast, for flow over a bump it is well established that there is only one solution in each case.

Although we have focused on the basic flow types 1-4, there is also the case of critical flow for which $F \neq 1$. Typically these flows are intrinsically unsteady and are sometimes referred to as transcritical flows [36-38]. However, in recent work, the present authors have demonstrated that steady flows over topography are possible under certain constraints and, moreover, they can be stable [39]. Binder et al. [40] considered the case of a finite-length corrugation for critical flow, $F=1$, using an inverse method. For an infinite (or semi-infinite) sinusoidal corrugation, it is straightforward to show that steady weakly nonlinear solutions are not possible under critical flow conditions. Setting $F=1$ and taking $\sigma=\cos (k x)$ in (8), we have $\eta_{x x}+(9 / 2) \eta^{2}=-3 \cos (k x)$. Integrating this equation over one period of the topography we obtain

$$
A+\frac{9}{2} \int_{0}^{2 \pi / k} \eta^{2} d x=0,
$$

where $A=\eta_{x}(2 \pi / k)-\eta_{x}(0)$. This forces $A<0$ and therefore $\eta_{x}$ must decrease monotonically in steps of one period of the topography; hence $\eta_{x} \rightarrow-\infty$ as $x \rightarrow \infty$ and a bounded solution is not possible. Given this failure for the weakly nonlinear model, it seems unlikely that steady solutions at $F=1$ exist for the fully nonlinear problem.

The hydraulic rise solutions we have computed may have practical relevance to hydraulic infrastructures, for example, in dams and sluice gates as was discussed in the Introduction. Of particular practical interest in these applications is the jump length and tailwater depth and how 
to reduce these. In such applications there may be a region of turbulent water across the jump, with a concomitant dissipation of energy [7-11]. Nevertheless, it is important to emphasize that we have shown that steady solutions do exist with a smooth, nonturbulent transition from one level to another. The issue of the stability of our solutions is an open question and is the subject of our ongoing investigations.

[1] J. T. Ellis, D. J. Sherman, B. O. Bauer, and J. Hart, Assessing the impact of an organic restoration structure on boat wake energy, J. Coastal Res. 36, 256 (2002).

[2] M. J. Bishop, A posteriori evaluation of strategies of management: The effectiveness of no-wash zones in minimizing the impacts of boat-wash on macrobenthic infauna, Environ. Manage. 34, 140 (2004).

[3] M. J. Bishop and M. G. Chapman, Managerial decisions as experiments: An opportunity to determine the ecological impact of boat-generated waves on macrobenthic infauna, Estuarine Coastal Shelf Sci. 61, 613 (2004).

[4] G. C. Nanson, A. Von Krusenstierna, E. A. Bryant, and M. R. Renilson, Experimental measurements of river-bank erosion caused by boat-generated waves on the Gordon River, Tasmania, River Res. Appl. 9, 1 (1994).

[5] A. J. Reynolds, Waves on the erodible bed of an open channel, J. Fluid Mech. 22, 113 (1965).

[6] A. G. Davies and A. D. Heathershaw, Surface-wave propagation over sinusoidally varying topography, J. Fluid Mech. 144, 419 (1984).

[7] S. Akib, A. A. Ahmed, H. M. Imran, M. F. Mahidin, H. S. Ahmed, and S. Rahman, Properties of hydraulic jump over apparent corrugated beds, Dam Eng. 25, 65 (2015).

[8] P. K. Yadav, Z. Ahmad, and G. L. Asawa, Parameters of hydraulic jump on corrugated beds, ISH J. Hydraul. Eng. 13, 93 (2007).

[9] M. M. Deshpande, V. R. Thombare, and S. D. Talegaonkar, Characteristics of hydraulic jump on corrugated beds, IRJET 3, 1764 (2016).

[10] S. Gandhi, Effect of corrugated bed on flow characteristics in rectangular open channel, WJTER 3, 350 (2018).

[11] S. A. Ead and N. Rajaratnam, Hydraulic jumps on corrugated beds, J. Hydraul. Eng. 128, 656 (2002).

[12] B. J. Binder, M. G. Blyth, and S. Balasuriya, Steady free-surface over spatially periodic topography, J. Fluid Mech. Rapids 781, R3 (2015).

[13] J.-M. Vanden-Broeck, Free-surface flow over an obstruction in a channel, Phys. Fluids 30, 2315 (1987).

[14] F. Dias and J.-M. Vanden-Broeck, Open channel flows with submerged obstructions, J. Fluid Mech. 206, 155 (1989).

[15] L. K. Forbes and L. W. Schwartz, Free-surface flow over a semicircular obstruction, J. Fluid Mech. 114, 299 (1982).

[16] L. K. Forbes, Non-linear, drag-free flow over a submerged semi-elliptical body, J. Eng. Math. 16, 171 (1982).

[17] F. Dias and J.-M. Vanden-Broeck, Generalised critical free-surface flows, J. Eng. Math. 42, 291 (2002).

[18] L. K. Forbes, Critical free-surface flow over a semi-circular obstruction, J. Eng. Math. 22, 3 (1988).

[19] E. O. Tuck, Ship-hydrodynamic free-surface problems without waves, J. Ship Res. 227, 227 (1991).

[20] F. Dias and J.-M. Vanden-Broeck, Trapped waves between submerged obstacles, J. Fluid Mech. 509, 93 (2004).

[21] T. R. Akylas, On the excitation of long nonlinear water waves by a moving pressure distribution, J. Fluid Mech. 141, 455 (1984).

[22] B. J. Binder and J.-M. Vanden-Broeck, Steady free-surface flow past an uneven bottom, Theor. Comput. Fluid Dyn. 20, 125 (2006).

[23] S. L. Cole, Transient waves produced by flow past a bump, Wave Motion 7, 579 (1985). 
[24] F. Dias and J.-M. Vanden-Broeck, Steady two-layer flows over an obstacle, Phil. Trans. R. Soc. Lond. A 360, 2137 (2002).

[25] R. Grimshaw and N. Smyth, Resonant flow of a stratified fluid over topography, J. Fluid Mech. 169, 429 (1986).

[26] S. J. Chapman and J.-M. Vanden-Broeck, Exponential asymptotics and gravity waves, J. Fluid Mech. 567, 299 (2006).

[27] C. J. Lustri, S. W. McCue, and B. J. Binder, Free-surface flow: A beyond all orders approach, Eur. J. Appl. Mech. 23, 441 (2012).

[28] B. J. Binder, M. G. Blyth, and S. W. McCue, Free-surface flow past arbitrary topography and an inverse approach to wave-free solutions, IMA J. Appl. Math. 78, 685 (2013).

[29] S. Balasuriya and B. J. Binder, Nonautonomous analysis of steady Korteweg-de Vries waves under nonlocalised forcing, Physica D 285, 28 (2014).

[30] J. Keeler, Free surface flow over bottom topography, Ph.D. thesis, University of Adelaide (2018).

[31] S. H. Strogatz, Nonlinear Dynamics and Chaos: With Applications to Physics, Biology, Chemistry, and Engineering (Westview Press, Boulder, 2014).

[32] B. J. Binder, J.-M. Vanden-Broeck, and F. Dias, Forced solitary waves and fronts past submerged obstacles, Chaos 15, 037106 (2005).

[33] B. J. Binder and M. G. Blyth, Electrified free-surface flow of an inviscid liquid past topography, Phys. Fluids 24, 102112 (2012).

[34] B. J. Binder and J.-M. Vanden-Broeck, The effect of disturbances on the free surface flow under a sluice gate, J. Fluid Mech. 576, 475 (2007).

[35] R. Chardard, F. Dias, H. Y. Nyguyen, and J.-M. Vanden-Broeck, Stability of some stationary soutions to the forced KdV equation with one or two bumps, J. Eng. Math. 70, 175 (2011).

[36] T. Y-T. Wu, Generation of upstream advancing solitons by moving disturbances, J. Fluid Mech. 184, 75 (1987).

[37] B. K. Ee, R. H. J. Grimshaw, D.-H. Zhang, and K. W. Chow, Steady transcritical flow over a hole: Parametric map of solutions of the forced Korteweg-de Vries equation, Phys. Fluids 22, 056602 (2010).

[38] R. Grimshaw, Transcritical flow past an obstacle, ANZIAM 52, 2 (2010).

[39] J. S. Keeler, B. J. Binder, and M. G. Blyth, On the critical free surface flow over bottom topography, J. Fluid Mech. 832, 73 (2017).

[40] B. J. Binder, M. G. Blyth, and S. Balasuriya, Non-uniqueness of steady free-surface flow at critical Froude number, EPL (Europhys. Lett.) 105, 44003 (2014). 